\title{
A Minimal Role for Selectins in the Recruitment of Leukocytes into the Inflamed Liver Microvasculature
}

\author{
John Wong, ${ }^{\star}$ Brent Johnston, ${ }^{\star}$ Samuel S. Lee, ${ }^{\star}$ Daniel C. Bullard,,$\|$ C. Wayne Smith, ${ }^{\ddagger}$ Arthur L. Beaudet, ${ }^{\S}$ and Paul Kubes ${ }^{\star}$ \\ *Immunology Research Group and Gastrointestinal Research Group, Department of Medicine and Department of Medical Physiology, \\ University of Calgary, Calgary, Alberta T2N 4N1, Canada; ${ }^{\circ}$ Department of Microbiology and Immunology, and ${ }^{\S}$ Department of \\ Molecular and Human Genetics, Baylor College of Medicine and Howard Hughes Medical Institute, Houston, Texas 77030; and \\ "Department of Comparative Medicine, University of Alabama at Birmingham, Birmingham, Alabama 35294
}

\begin{abstract}
A two-step paradigm for leukocyte recruitment has been established in a number of tissues including the mesentery, skin, and muscle, and necessitates an initial rolling step via the selectins before firm leukocyte adhesion via the integrins. In view of the many inflammatory diseases that involve the liver, we investigated the importance of rolling and the selectins in the hepatic microvasculature and compared the responses to that of the commonly used mesentery or cremaster microvasculature. We visualized the liver microvasculature using intravital microscopy and we determined that within the liver the majority of leukocytes adhere within the sinusoids $(80 \%)$ in response to a chemotactic stimulus such as FMLP (20\% in postsinusoidal venules) whereas leukocytes adhere exclusively within postcapillary venules in tissue like the mouse cremaster. In the sinusoids, the adhesive response to FMLP is not dependent upon selectins inasmuch as adhesion was not reduced in the sinusoidal vessels of P-selectin-deficient mice or E-selectin/P-selectindeficient animals in the presence or absence of L-selectin antibody. No rolling or adhesion was detected in response to FMLP in the selectin-deficient cremaster microvasculature. Immunoneutralization of selectins with fucoidan in wildtype mice eliminated rolling and adhesion in the cremaster but failed to affect adhesion in the liver sinusoids in response to FMLP. More long-term leukocyte recruitment with lipopolysaccharide $(4 \mathrm{~h})$ was also impaired in the cremaster but not the liver microvasculature in selectin-deficient animals. Leukocyte adhesion in the sinusoids was reduced in P-selectin-deficient mice also lacking intercellular adhesion molecule-1 (ICAM-1). This study for the first time demonstrates that selectins are not an essential step for leukocyte recruitment into the inflamed liver microvasculature. $(J$. Clin. Invest. 1997. 99:2782-2790.) Key words: sinusoids • inflammation • rolling • adhesion
\end{abstract}

Address correspondence to Dr. Paul Kubes, Immunology Research Group, Department of Medical Physiology, Faculty of Medicine, University of Calgary, Calgary, Alberta T2N 4N1, Canada. Phone: 403-220-8558; FAX: 403-283-1267; E-mail: pkubes@acs.ucalgary.ca

Received for publication 19 December 1996 and accepted in revised form 14 March 1997.

J. Clin. Invest.

(C) The American Society for Clinical Investigation, Inc.

0021-9738/97/06/2782/09 \$2.00

Volume 99, Number 11, June 1997, 2782-2790

\section{Introduction}

Leukocyte recruitment into tissues is an essential defense mechanism in the body's armament against invading pathogens. Visualization of leukocyte behavior within inflamed vessels of translucent tissues such as the mesentery (1-3) or cremaster $(4,5)$ has revealed that leukocytes tether to the endothelial lining and roll along the length of the venule before firmly adhering to the vasculature. These experiments, performed on $20-40-\mu \mathrm{m}$ postcapillary venules, have revealed that the rolling event is an important prerequisite for adhesion and migration into tissues and has produced the two-step paradigm for leukocyte recruitment: leukocyte rolling being the initial step followed by leukocyte adhesion (6-8). Interestingly, the two-step paradigm has not been examined in organs like the liver, which is a venous driven vascular bed with slow flow rates. Therefore, leukocyte recruitment in this organ may or may not behave like the mesentery or cremaster muscle. Defining the mechanisms underlying leukocyte recruitment in the liver is extremely important not just for our understanding of the inflammatory process in the liver, but also in directing our attempts to design antiadhesion therapies to reduce hepatic inflammation.

As is the case in other organs, neutrophils appear to contribute significantly to liver injury in experimental models of endotoxin shock and ischemia/reperfusion $(9,10)$. Published data suggest an important role for adhesion molecules inasmuch as immunoneutralization of intercellular adhesion molecule-1 (ICAM-1) ${ }^{1}$ or $\beta_{2}$-integrin (CD11a or CD11b) with monoclonal antibodies decreased inflammation-induced liver injury $(11,12)$. Moreover, a role for selectins has also been invoked based on the fact that various inflammatory stimuli induce rapid expression of $\mathrm{P}$-selectin: increased $\mathrm{E}$ - and $\mathrm{P}$-selectin mRNA in liver endothelial cells and increased expression of P-selectin and E-selectin within the liver microvasculature, consistent with an involvement for selectins in neutrophil rolling in the liver microvasculature (13-16). However, a direct assessment (visualization) of the importance of selectin-dependent leukocyte rolling in the inflamed liver microcirculation has not been documented to date.

Therefore, our objective was to assess the multistep recruitment paradigm in the liver microvasculature in animals lacking P-selectin, both endothelial selectins (E-selectin/P-selectindeficient mice), and combined P-selectin and ICAM-1. We report that the majority of leukocytes adhere in liver sinusoids (equivalent of capillaries) rather than in the postcapillary venules in response to a chemotactic stimulus. There is an absence of rolling but not adhesion in these specialized microves-

1. Abbreviations used in this paper: ICAM-1, intercellular adhesion molecule-1; MPO, myeloperoxidase. 
sels and the adhesion appears to entirely bypass the selectins inasmuch as mice lacking P-selectin or P-selectin and E-selectin in the presence or absence of functional L-selectin displayed identical adhesion in the sinusoids to their wild-type counterparts. Deletion of P-selectin and ICAM-1, however, prevented adhesion in sinusoids associated with inflammation, suggesting a role for adhesion molecules in leukocyte-endothelial cell interactions in the liver. This study demonstrates that selectins are not an essential step for leukocyte recruitment into the inflamed liver microvasculature.

\section{Methods}

Intravital microscopy in mouse cremasteric venules. Mice deficient in P-selectin or both P-selectin and E-selectin were generated by gene targeting in embryonic stem cells as described previously $(17,18)$. The mice were from a mixed background of $129 \mathrm{~Sv} \times \mathrm{C} 57 \mathrm{Bl} / 6$. As these controls were not different from $\mathrm{C} 57 \mathrm{Bl} / 6$ mice, in some experiments the latter were used and controls were grouped together. All animals were maintained on purified laboratory diet in specific pathogen-free facilities. Animals were anesthetized with a mixture of ketamine and xylazine administered intraperitoneally (200 and 10 $\mathrm{mg} / \mathrm{kg}$, respectively) and prepared for intravital microscopy. Briefly, the right jugular vein was cannulated for maintenance of anesthesia during the experiment. The cremaster muscle was dissected free of tissues and exteriorized onto an optically clear viewing pedestal. The muscle was cut longitudinally with a cautery and held flat against the pedestal by attaching silk sutures to the corners of the muscle. The muscle was then superfused with bicarbonate-buffered saline. This preparation has been used extensively to study leukocyte-endothelial cell interactions within the murine microcirculation $(4,18)$.

Intravital microscopy in mouse hepatic microvasculature. After identical anesthetic and cannulating procedures as described above, mice were prepared for intravital microscopy of the liver microcirculation. A midline and a left subcostal incision were made in order to exteriorize the liver. The hepatic ligaments were dissected and the intestine was covered with a moist gauze. Animals were placed in a left supine position and the left liver lobe was positioned onto a Plexiglas microscopic stage as described in detail previously $(19,20)$. The liver surface was then covered with Saran Wrap to hold the organ in position.

Intravital microscopy in rat mesentery and liver. Male SpragueDawley rats $(180-250 \mathrm{~g})$ were maintained on a purified laboratory diet and fasted for $24 \mathrm{~h}$ before surgery. The animals were anesthetized with pentobarbital sodium intraperitoneally $(65 \mathrm{mg} / \mathrm{kg}$ body wt). The right carotid artery was cannulated for measurement of systemic arterial blood pressure using a Statham P23XL pressure transducer (Viggo-Spectramed, Oxnard, CA) and Grass physiologic recorder (Grass Instrument Co., Quincy, MA). A catheter was placed in the right jugular vein for administration of drugs and additional anesthesia. A midline laparotomy was made and the animals were placed in a supine position on an adjustable Plexiglas microscopic stage. A segment of midjejunum was exteriorized gently and all exposed tissue was covered with saline-soaked gauze to minimize tissue dehydration. The mesentery was then placed over an optically clear viewing pedestal that allowed for transillumination of a $2-\mathrm{cm}^{2} \mathrm{seg}-$ ment of tissue. The temperature of the pedestal was maintained at $37^{\circ} \mathrm{C}$ with a constant temperature water circulator (Haake Fisons, Karlsruhe, Germany) and the animals were kept at $37^{\circ} \mathrm{C}$ using an infrared heat lamp. Rectal temperature was monitored using an electrothermometer. The exposed mesentery was suffused with warm bicarbonate-buffered saline ( $\mathrm{pH}$ 7.4) using a peristaltic pump (Gilson Minipuls3; Villiers, Le Bel, France), while excess fluid was removed via a suction pump. This preparation has been used extensively by us and others to study leukocyte-endothelial cell interactions within the mesenteric circulation $(1,8,21,22)$.

To study the rat liver microcirculation, the same anesthetic was used as described for the rat mesentery, and the same surgical preparation was used as described for mouse liver.

Leukocyte parameters. Initially, animals received rhodamine $6 \mathrm{G}$ intravenously $(0.3 \mathrm{mg} / \mathrm{kg}$ body wt) to label leukocytes as previously described (23). Rhodamine 6G-associated fluorescence was visualized by epi-illumination at $510-560 \mathrm{~nm}$, using a $590 \mathrm{~nm}$ emission filter. As this agent did not affect leukocyte kinetics, and transillumination could be used for the mouse cremaster and rat mesentery, rhodamine $6 \mathrm{G}$ was used primarily in the liver preparations.

An intravital microscope (Optiphot-2; Nikon Inc., Mississauga, Canada) with a $\times 25$ objective lens (L25/0.35; E. Leitz Inc., Munich, Germany) and a $\times 10$ eyepiece was used to examine the mesenteric microcirculation and a $\times 40$ water immersion lens $(40 / 0.55 \mathrm{WI}$; Nikon, Tokyo, Japan) was used to observe the microcirculatory events on the surface of the liver. A silicon-intensified fluorescent camera (model C-2400-08; Hamamatsu Photonics, Hamamatsu City, Japan) mounted on the microscope projected the image onto a monitor and the images were recorded for playback analysis using a videocassette recorder. The number of rolling and adherent leukocytes was determined offline during video playback analysis. Leukocytes were considered adherent to the venular endothelium if they remained stationary for a period of time $\geq 30 \mathrm{~s}$. Rolling leukocytes were defined as those moving at a velocity less than that of erythrocytes within a given vessel. The number of rolling leukocytes along the endothelial lining was also recorded. After the liver was isolated and placed under the intravital microscope, the centrilobular zones were located. Within each field of view $\left(2.1 \times 10^{4} \mu \mathrm{m}^{2}\right) \sim 8-10$ centrilobular sinusoids were observed, each of them emptying into a terminal hepatic venule (postsinusoidal venule). Sinusoids and hepatic venules were identified by their diameters and directions of blood flow. In a single animal, 10-15 acinar zones were studied. Mesenteric and cremaster venules of 20 $40 \mu \mathrm{m}$ diameter were selected for study and the same section of the venule was observed throughout the experiment.

Experimental protocols. We examined the role of selectins in leukocyte recruitment in P-selectin-deficient mice, E-selectin/P-selectindeficient and E-selectin/P-selectin-deficient mice treated with an L-selectin monoclonal antibody (Mel-14, rat anti-mouse, $3 \mathrm{mg} / \mathrm{kg}$ body wt, administered intravenously; PharMingen, San Diego, CA) as well as wild-type animals. The chemotactic peptide FMLP (Sigma Chemical Co., St. Louis, MO; $10 \mu \mathrm{M}$ ) was superfused onto the mouse liver or cremaster microcirculation for $60 \mathrm{~min}$, and recordings were made every $15 \mathrm{~min}$. In another series of experiments exploring the role of selectins, mice received $10 \mathrm{mg} / \mathrm{kg}$ of the selectin binding carbohydrate fucoidan and either the cremaster or liver was again superfused with FMLP $(10 \mu \mathrm{M})$. To test the possibility that other adhesion molecules aside from selectins were important in the liver microvasculature, we compared the adhesion of leukocytes in P-selectin-deficient animals directly with P-selectin/ICAM-1-deficient animals. It should be noted that the ICAM- 1 mutant mice express $\sim 1 \%$ of the ICAM-1 found in a normal or P-selectin-deficient animal (24). In a final series of experiments, instead of FMLP, animals were injected with lipopolysaccharide (LPS) $(50 \mu \mathrm{g}$ per mouse, administered intraperitoneally; Sigma Chemical Co.) and $4 \mathrm{~h}$ later the liver or cremaster was exteriorized as described above. Leukocyte rolling and adhesion was again documented

In rat experiments, the mesentery or liver was superfused with FMLP $(5 \mu \mathrm{M})$ in the presence or absence of fucoidan $(25 \mathrm{mg} / \mathrm{kg}$; Sigma Chemical Co.) as described previously $(8,25)$.

Liver biochemistry. Alanine aminotransferase was measured using commercially available Sigma Chemical Co. diagnostic kits. The enzyme was measured in sera that had been frozen for a maximum of 2 wk.

Determination of tissue myeloperoxidase (MPO) activity. Samples of liver were washed extensively, weighed, frozen on dry ice, and processed for determination of MPO activity. MPO is an enzyme found in cells of myeloid origin, and has been used extensively as a biochemical marker of granulocyte (mainly neutrophil) infiltration into tissues. The samples were stored at $-20^{\circ} \mathrm{C}$ and the MPO activity was 
determined using an assay described previously (26), but with the volumes of each reagent modified for use in a 96-well enzyme-linked immunosorbent assay plate. Change in absorbance at $450 \mathrm{~nm}$ over a $90-\mathrm{s}$ period was determined using a kinetic microplate reader (Molecular Devices, Canada)

Statistical analysis. All values are reported as means \pm SEM. Differences between the groups were assessed by one-way ANOVA followed by a Bonferroni correction for multiple comparisons. Statistical significance was set at $P<0.05$.

\section{Results and Discussion}

Previous work has clearly demonstrated that chemotactic factors (platelet activating factor, leukotriene $\mathrm{B}_{4}, \mathrm{FMLP}$ ) as well as cytokines or LPS will induce leukocyte infiltration into postcapillary venules of the rat $(8,27)$, rabbit $(6,28)$, and cat mesentery $(29,30)$, the mouse cremaster $(4,18,19)$, and the hamster cheek pouch (31-33). Intravital microscopy reveals that

A

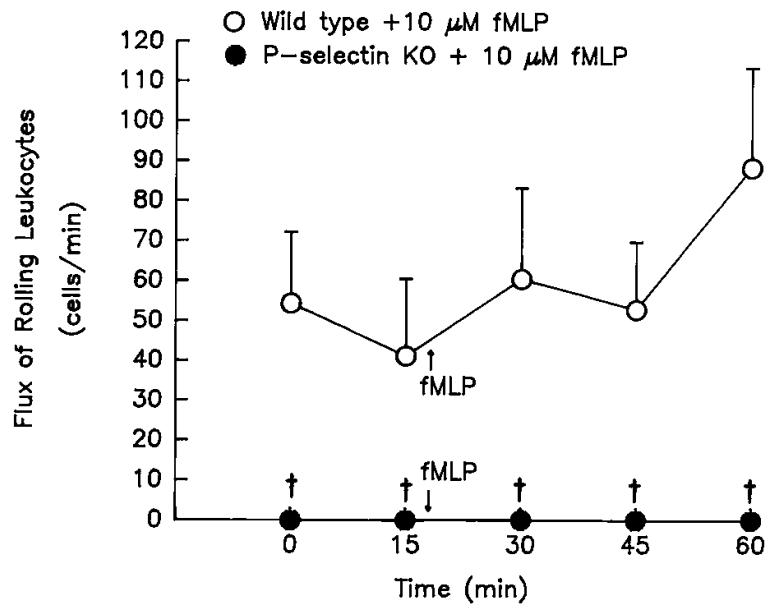

B

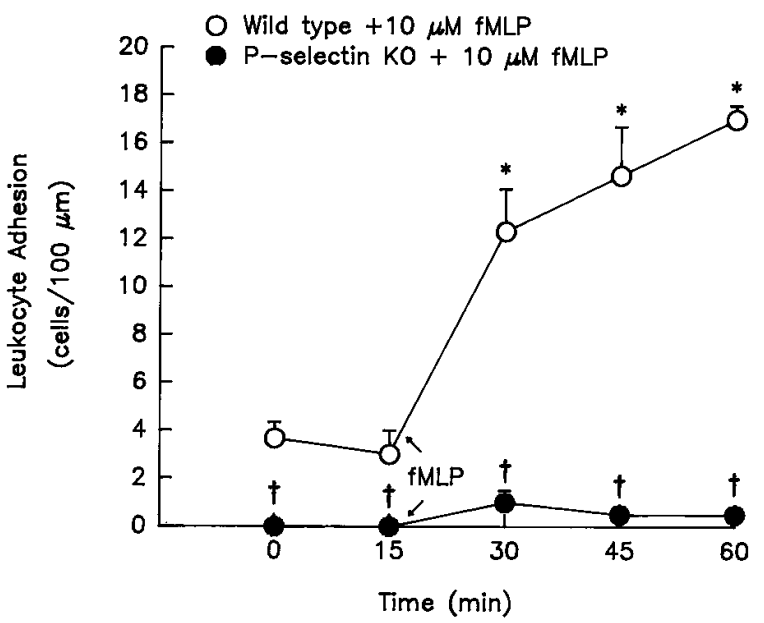

Figure 1. Leukocyte rolling flux and adhesion in cremasteric venules superfused with FMLP. After an initial 20-min control period during which preparations were superfused with bicarbonate-buffered saline, cremasteric preparations of wild-type $(O)$ and P-selectin-deficient $(\bullet)$ mice were superfused continuously with $10 \mu \mathrm{M}$ FMLP. $(A)$ Flux of rolling leukocytes, and $(B)$ leukocyte adhesion were measured at 15 -min intervals. Data are represented as means \pm SEM. $* P<$ 0.05 versus time 0 min untreated period. ${ }^{\dagger} P<0.05$ versus wild-type; $n=7$. the mouse cremaster microvasculature displays some rolling but little or no adhesion under control conditions. Exposure of the mouse cremaster to the chemotactic factor FMLP induces a rapid increase in leukocyte adhesion in postcapillary venules of wild-type mice (Fig. 1). It is noteworthy that adhesion only occurred in postcapillary venules; there was absolutely no adhesion in any of the other microvascular compartments including the capillaries and arterioles (not shown). Animals lacking $\mathrm{P}$-selectin had absolutely no rolling or adhesion in cremaster microcirculation in response to FMLP (Fig. 1). Clearly, rolling is essential for subsequent adhesion in cremaster muscle vasculature in response to an inflammatory process.

Although the leukocyte recruitment cascade has been postulated as a universal concept, the hypothesis has only been tested (visualized) in the microvasculature of translucent tissues. The general recruitment cascade has not been studied systematically in organs such as the liver. Visualization of the liver reveals an extensive capillary network (sinusoids) with slow flow rates that drain into postsinusoidal venules. Fig. $2 \mathrm{~A}$ demonstrates that leukocyte adhesion occurred in both the sinusoids and postsinusoidal venules in response to the chemotactic factor FMLP. Moreover, leukocyte adhesion in the sinusoids accounted for $70-80 \%$ of the total number of adherent
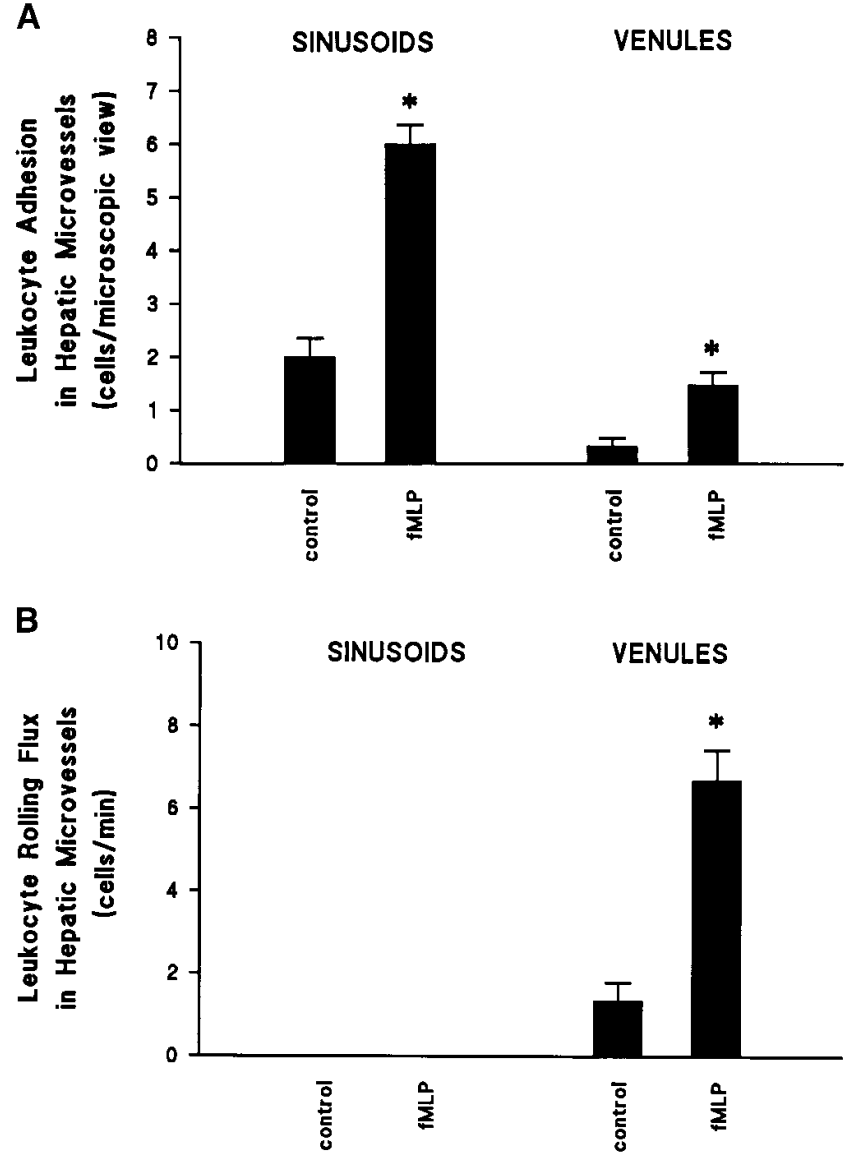

Figure 2. Leukocyte adhesion and rolling flux in sinusoids and hepatic venules superfused with FMLP. Preparations were superfused continuously with bicarbonate-buffered saline (control) or $10 \mu \mathrm{M}$ FMLP. $(A)$ Leukocyte adhesion and $(B)$ rolling flux were determined after 60 min of treatment. Data are represented as means \pm SEM. $* P<$ 0.05 versus control; $n=8$. 

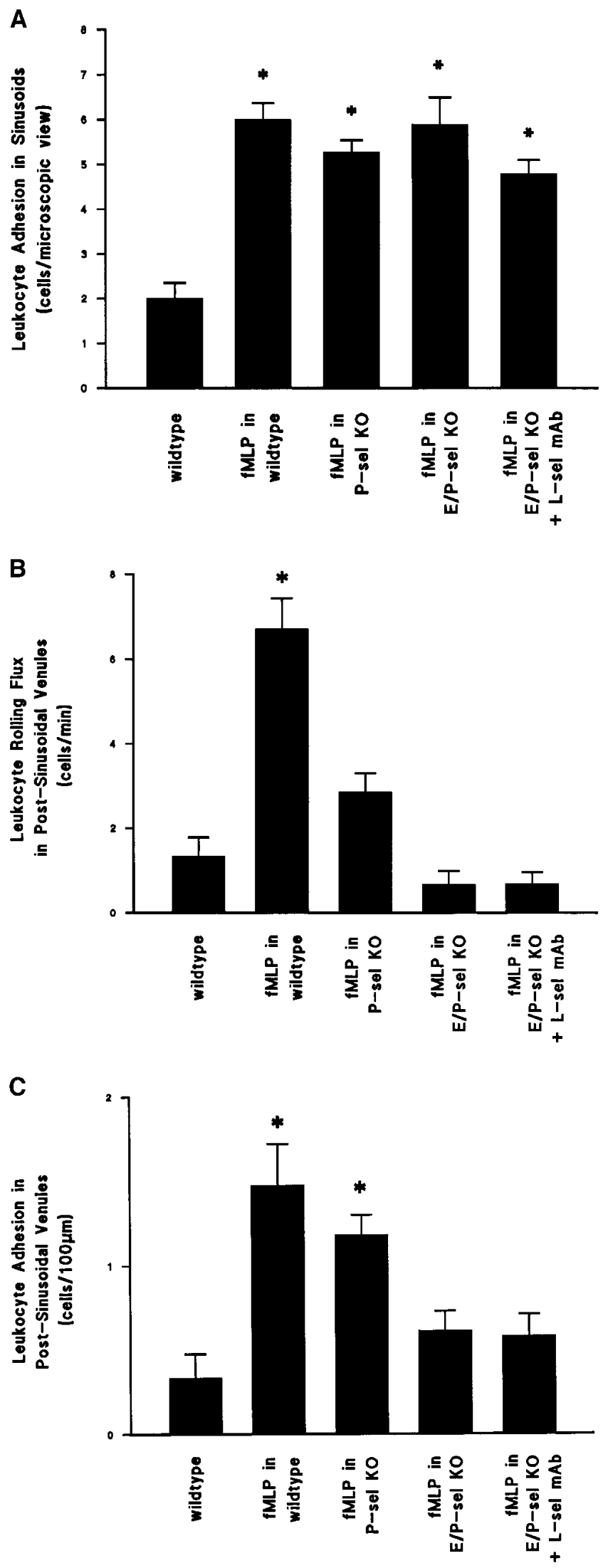

Figure 3. The role of selectins in leukocyte adhesion and rolling flux in sinusoids and hepatic venules. Liver preparations were studied in wild-type, P-selectin-deficient, and double E-selectin/P-selectindeficient mice. To eliminate contributions of all three selectins, some cells within the liver microcirculation. However, rolling leukocytes, defined as those cells moving slower than the red blood cells with detectable rotational motion, could only be seen in the postsinusoidal venules, not in the sinusoids per se (Fig. $2 \mathrm{~B})$. Administration of FMLP increased leukocyte rolling in the postsinusoidal venules but rolling was still not detectable in the sinusoids (Fig. 2 B).

Although these data would suggest that $80 \%$ of leukocytes in the inflamed liver microvasculature adhere independent of a rolling motion, it is possible that selectins may still have a functional role in the capturing/tethering of leukocytes within the sinusoidal compartment of the liver microcirculation. Fig. $3 A$ demonstrates that leukocyte adhesion in sinusoids occurred as effectively in P-selectin-deficient animals as wildtype littermates in response to FMLP. Because there is good evidence that the selectins have overlapping functions, particularly in the single adhesion molecule knockout mice (18, 34, 35), we tested animals deficient in both E-selectin and P-selectin. Fig. $3 \mathrm{~A}$ demonstrates that mice deficient in both P-selectin and E-selectin still had leukocyte adhesion in sinusoidal vessels in response to FMLP. This is in sharp contrast to previous work that demonstrates that this double deletion prevents recruitment of leukocytes into the peritoneal cavity, cremasteric circulation, or skin in response to numerous inflammatory conditions $(18,36)$. For completeness, some of the E-selectin/ $\mathrm{P}$-selectin knockout mice received an L-selectin antibody at concentrations that reduced leukocyte rolling in other vascular beds (4); leukocyte adhesion was not affected in the sinusoidal vessels.

Fig. 3, $B$ and $C$, illustrates that the rise in leukocyte rolling and adhesion in the postsinusoidal venules was modest and returned to control in mice deficient in both E-selectin and P-selectin regardless of whether the L-selectin antibody was added. Interestingly, there was some residual rolling in P-selectin-deficient postsinusoidal vessels (but not cremaster muscle) which permitted leukocytes to adhere in these vessels. This low level of baseline rolling may be related to constitutive E-selectin expression (rolling not present in E-selectin/P-selectin knockout mice) perhaps as a result of continuous translocation of noxious stimuli from the intestinal lumen.

On rare occasions a cell could be seen rolling and then adhering in the postsinusoidal venules of all mice including E-selectin/P-selectin knockout mice. This may reflect the low shear forces in the postsinusoidal venules. Indeed, we (25) have demonstrated previously that leukocyte rolling and subsequent adhesion were entirely inhibited in rat mesenteric postcapillary venules by the selectin-binding carbohydrate fucoidan. If shear was lowered by $50 \%$, leukocytes began to roll and adhere despite the presence of fucoidan. This increase in rolling and adhesion was entirely dependent on $\beta_{2}$-integrins and may also underlie the adhesion in liver postsinusoidal venules of the selectin-deficient animals.

E-selectin/P-selectin-deficient animals were also given an anti-L-selectin $\mathrm{mAb}$ intravenously (Mel-14, $3 \mathrm{mg} / \mathrm{kg}$ ). Preparations were superfused continuously with bicarbonate-buffered saline alone or $10 \mu \mathrm{M}$ FMLP. $(A)$ Leukocyte adhesion was determined in the sinusoids; and $(B)$ leukocyte rolling flux and $(C)$ adhesion were determined in the postsinusoidal venules $60 \mathrm{~min}$ after FMLP treatment. Data are represented as means \pm SEM. $* P<0.05$ versus untreated wild-type; $n=18$. 


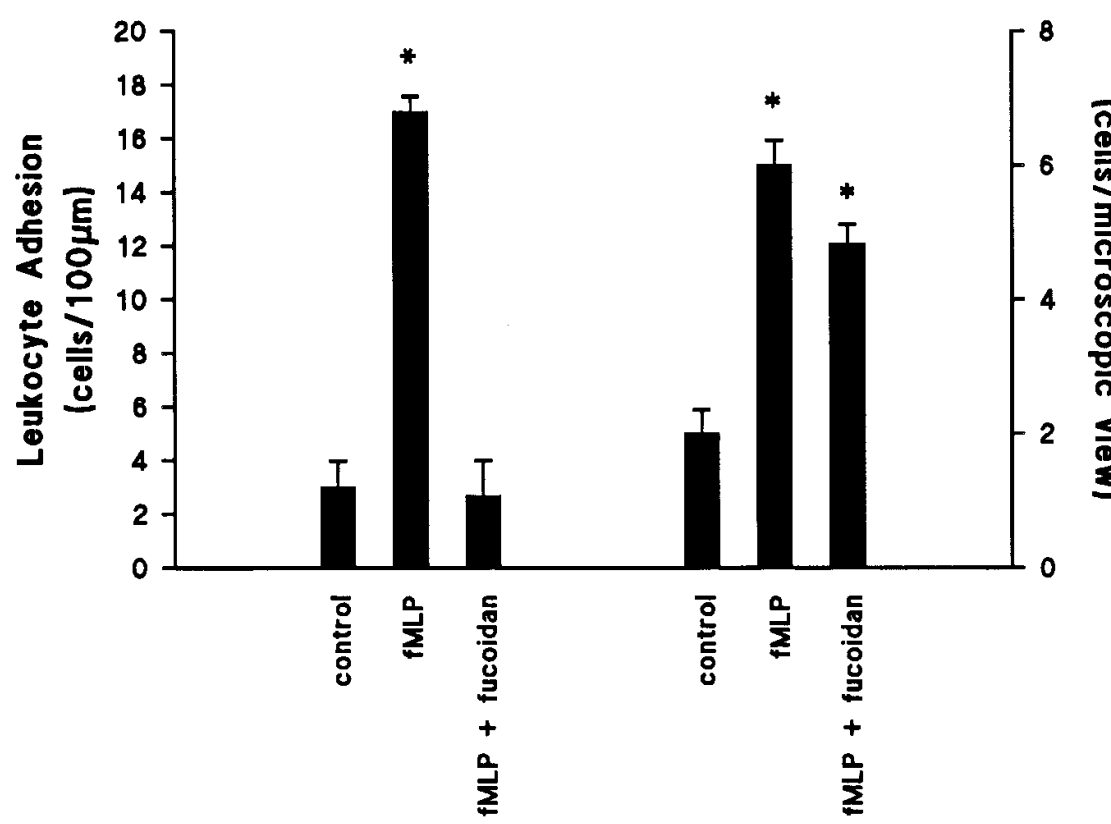

Figure 4. The effects of fucoidan on FMLPinduced leukocyte adhesion in mouse cremasteric venules and hepatic sinusoids. The selectin-blocking polysaccharide fucoidan $(10 \mathrm{mg} /$ $\mathrm{kg}$ ) was administered intravenously before superfusion of the preparation with $10 \mu \mathrm{M}$ FMLP. Leukocyte adhesion was determined after 60 min of FMLP treatment. Data are represented as means \pm SEM. $* P<0.05$ versus untreated control; $n=11$.
A criticism often levied against the use of mutant (genedeleted) mice is that there may be redundancy in these animals. Certainly, one possibility to explain the E-selectin/P-selectin data in the hepatic microvasculature is to invoke the induction of another as yet unidentified adhesion molecule. To test the possibility that selectins were important in the hepatic circulation of wild-type mice, some animals received the selectinbinding carbohydrate fucoidan, which essentially abolishes rolling in the mouse system $(<5$ cells rolling per min in the cremaster microvasculature), and examined adhesion in the cremaster and liver microcirculation. The results shown in Fig. 4 revealed that immunoneutralization of selectins abolished adhesion in the cremasteric venules but failed to inhibit leukocyte adhesion in hepatic sinusoids. As this response is identical to that observed in the selectin-deficient animals, it suggests that the selectin-independent adhesion is manifested in all mice (not just the mutant animals).

Finally, to ensure that the selectin-independent adhesion in sinusoids was not restricted to mice, we examined the same principle in rats. Fig. 5 demonstrates that the rise in leukocyte
MESENTERY

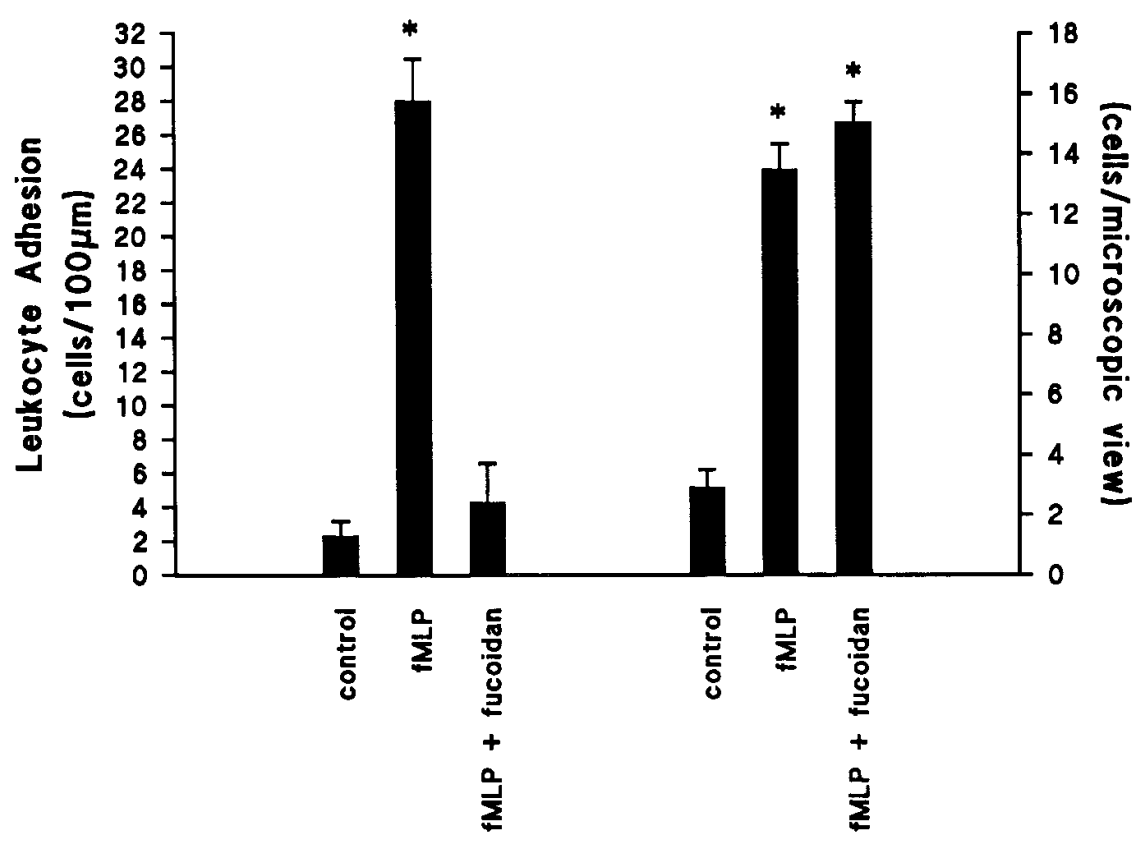

Figure 5. The effect of fucoidan on FMLPinduced leukocyte adhesion in rat mesenteric venules and hepatic sinusoids. The selectinblocking polysaccharide fucoidan $(25 \mathrm{mg} / \mathrm{kg})$ was administered intravenously before superfusion of the preparation with $10 \mu \mathrm{M}$ FMLP. Leukocyte adhesion was determined after 60 min of FMLP treatment. Data are represented as means \pm SEM. $* P<0.05$ versus untreated control; $n=12$. 


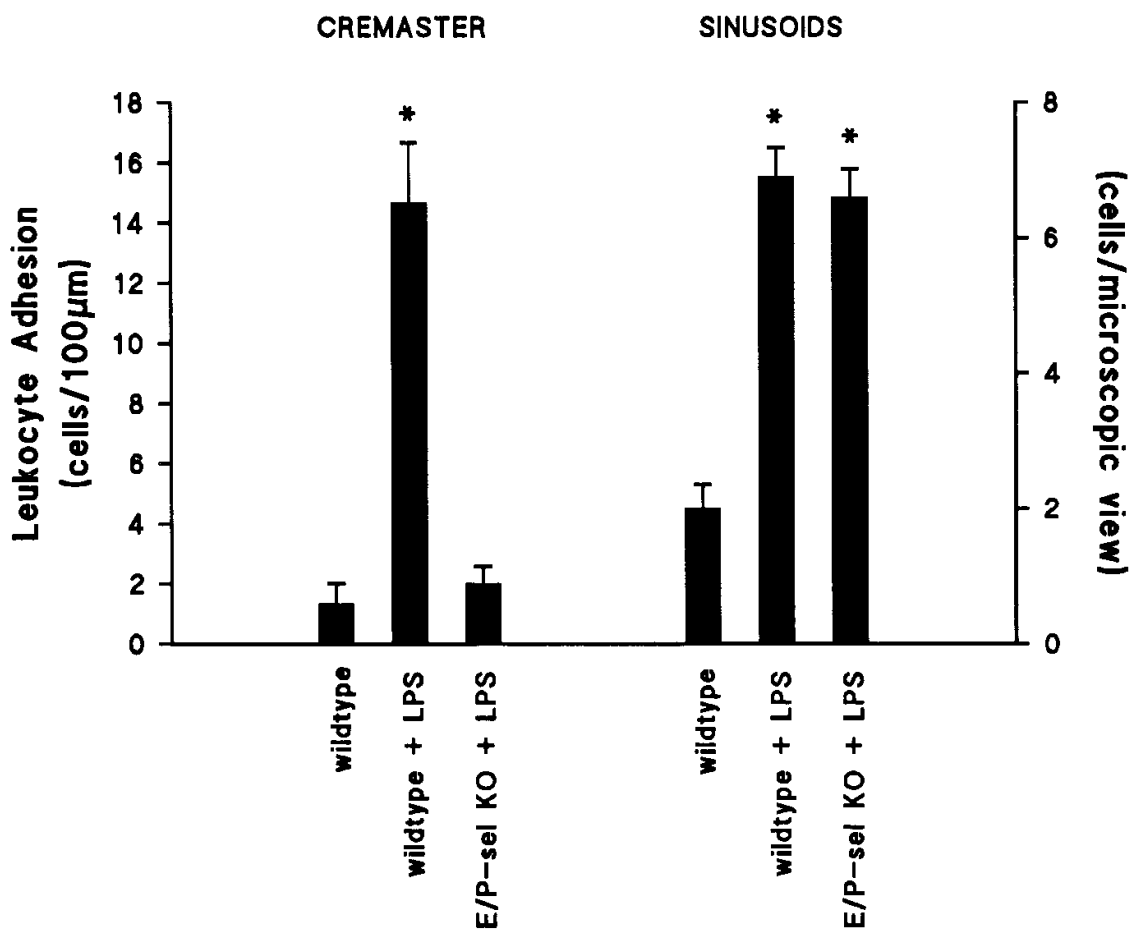

Figure 6. Leukocyte adhesion in mouse cremasteric venules and hepatic sinusoids after LPS administration. LPS $(50 \mu \mathrm{g})$ was administered intraperitoneally to wild-type and E-selectin/P-selectin-deficient mice. Leukocyte adhesion in cremasteric venules and hepatic sinusoids was determined $4 \mathrm{~h}$ later. Data are represented as means \pm SEM. $* P<0.05$ versus control; $n=10$. adhesion in response to FMLP was entirely eliminated in the rat mesentery when leukocyte rolling was prevented with fucoidan. However, leukocyte adhesion within the rat liver sinusoids was not affected by the selectin-binding carbohydrate.

To confirm that the selectin-independent adhesion in sinusoids was not restricted to FMLP, LPS, a stimulus that causes synthesis of adhesion molecules including E-selectin, was tested. The data reveal that regardless of stimulus, selectins were not necessary for adhesion in the liver microcirculation inasmuch as LPS induced significant adhesion in the E-selectin/P-selectin-deficient liver microvasculature (Fig. 6). By contrast, LPS was not able to induce adhesion in the E-selectin/ P-selectin-deficient cremaster microvasculature. LPS-induced liver injury has been shown to be dependent upon neutrophils previously $(10,11)$. In this study, a threefold increase in liver enzyme levels (alanine aminotransferase, ALT) was noted in E-selectin/P-selectin knockout mice (Fig. $7 A$ ) and their wildtype littermates in response to LPS. This is consistent with the view that the selectins were a prerequisite for neither $(a)$ leukocyte infiltration into the liver microcirculation, nor $(b)$ liver injury. MPO activity was also measured in these animals (Fig. $7 \mathrm{~B}$ ), and these values were the same in the E-selectin/ $\mathrm{P}$-selectin knockout mice and wild-type animals in response to LPS.

It is intriguing that in this study we observed the same number of adhering cells in normal animals and animals deficient in P-selectin and P-selectin/E-selectin despite the fact that the circulating levels of leukocytes were seven- to eightfold higher in the P-selectin/E-selectin-deficient animals than their wildtype counterparts. Although one could argue that this may support a role for selectins in this model, this argument presumes that the number of adherent cells will be proportional to the number of rolling and circulating leukocytes. We have already demonstrated that only a fraction of rolling cells ad- here, i.e., one must inhibit $90 \%$ of rolling cells to impact on the magnitude of adhesion (2). In other words, it is the number of adhesive sites that regulates the magnitude of adherent cells and if these sites are saturated, larger numbers of rolling leukocytes will not increase adhesion. An identical relationship may exist for circulating versus adherent cells; inflamed vessels recruit only a fraction of the circulating pool and one has to deplete a very significant number of circulating leukocytes (95$100 \%$ ) before leukocyte adhesion and infiltration are affected (37). It is conceivable that high levels of circulating cells could impact on tissue levels of leukocytes in models of frank hemorrhage where adhesion may not be the only mechanism by which leukocytes are delivered to tissues (Kubes, P., unpublished observations); however in this study, bleeding was not observed.

It is clear from the many studies completed in postcapillary venules of tissues such as the mesentery, cremaster, and cheek pouch that leukocytes tether and roll via the selectins and firmly adhere via the integrins on the endothelial lining. However, more specialized vascular beds such as the liver microcirculation have a low flow state with high capillary density. Under these conditions, it is clear that leukocytes can adhere in the sinusoids entirely independent of either of the two endothelial selectins. Moreover, immunoneutralization of all three selectins in the same animal still did not prevent leukocyte recruitment into the liver. These data clearly challenge the generalized concept of the multistep leukocyte recruitment requiring selectin-dependent rolling as a prerequisite for integrin-dependent adhesion in the liver microvasculature. As $80 \%$ of the infiltrating leukocytes adhered within the sinusoids, the majority of cells were clearly infiltrating the liver independent of selectins.

As the sinusoids are small vessels with discontinuous endothelial lining, adhesion may be entirely independent of en- 

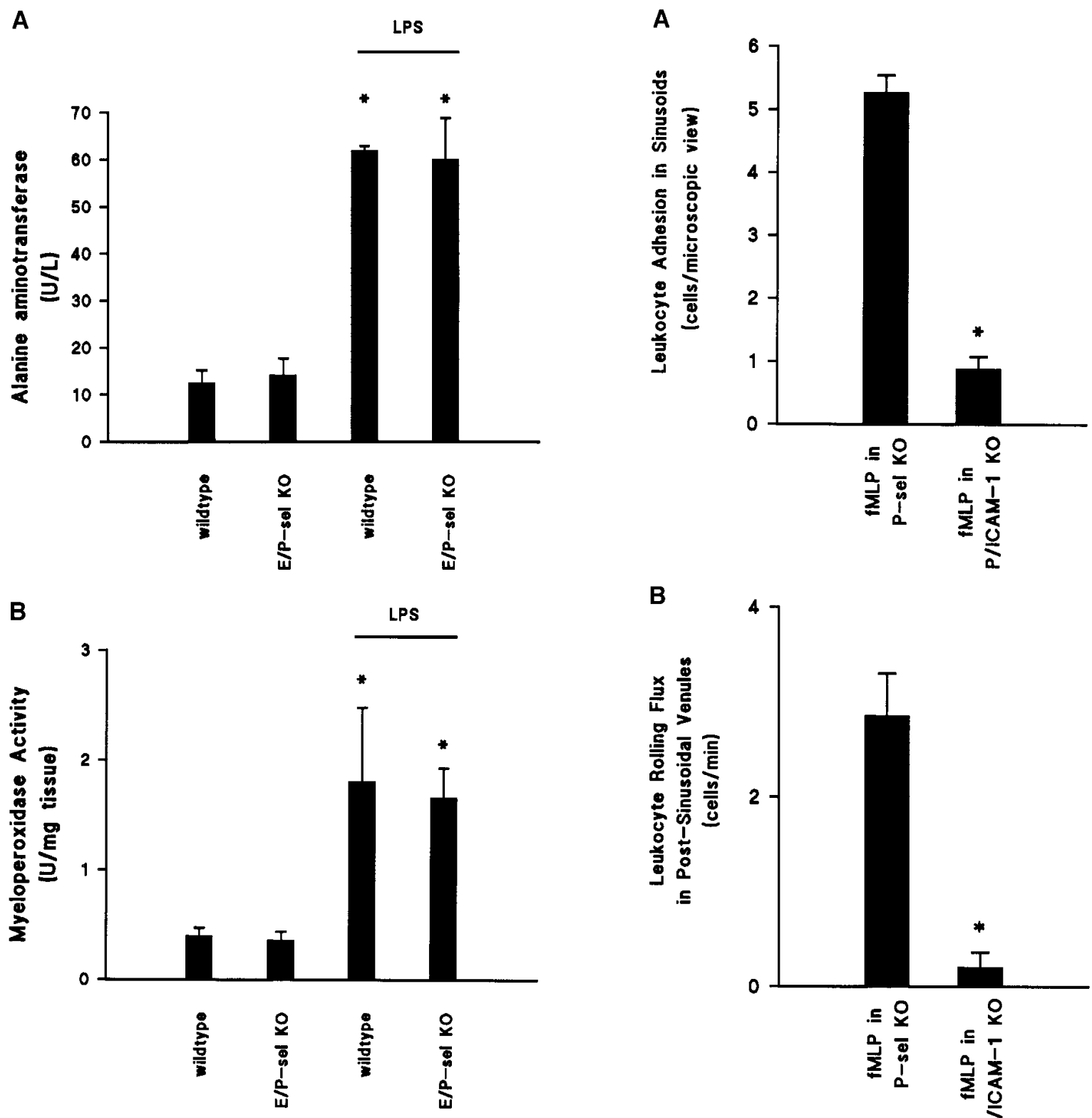

Figure 7. Alanine aminotransferase levels $(A)$ and MPO activities $(B)$ in the experimental groups after $4 \mathrm{~h}$ of LPS. Data are represented as means \pm SEM. $* P<0.05$ versus control; $n=11$.

dothelial adhesion molecules. To test this possibility, we directly compared the leukocyte adhesion in the sinusoids of P-selectin-deficient animals with P-selectin-deficient animals that also lacked ICAM-1. Fig. 8 A demonstrates that ICAM-1 is indeed essential for leukocyte adhesion within the sinusoids and that mechanical trapping of leukocytes within these microvessels (as has been proposed for the lung) is clearly not the mechanism by which the cells adhere in liver in response to FMLP. Fig. $8 B$ shows that leukocyte rolling and adhesion

Figure 8. The role of ICAM-1 in leukocyte adhesion and rolling flux in hepatic microvasculature. $(A)$ Leukocyte adhesion in sinusoids; $(B)$ leukocyte rolling and $(C)$ adhesion in postsinusoidal venules. Data are represented as means \pm SEM. $* P<0.05$ versus control; $n=6$.

B

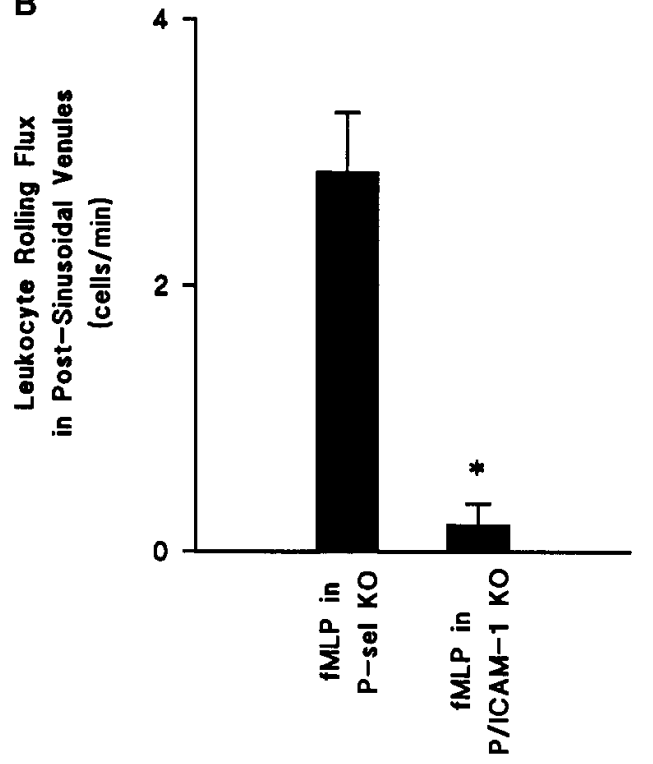

C

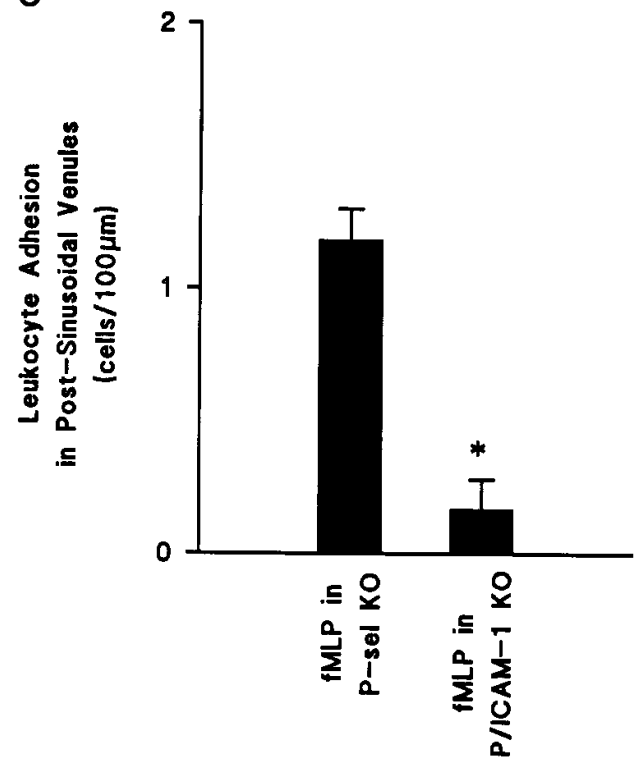


within FMLP-treated postcapillary venules were also entirely inhibited within postsinusoidal venules lacking P-selectin and ICAM-1. It is noteworthy that the P-selectin knockout mice still had rolling and adhesion in these vessels. This observation is consistent with previous work by Kunkel et al., who demonstrated less rolling in P-selectin/ICAM-1-deficient mice than in P-selectin-deficient mice (38), and with our own hypothesis that at lower shear forces (as is the case in these postcapillary venules), leukocyte rolling and adhesion can occur via nonselectin pathways (25).

Therefore, these data, for the first time, suggest that antiselectin therapy, with the aim of preventing leukocyte rolling and thereby reducing leukocyte adhesion, is unlikely to impact upon the inflammatory process within the liver per se. First, in systemic inflammatory conditions such as trauma-induced shock leading to multiple organ failure, interrupting the inappropriate inflammatory response with antiselectin therapy may have little effect on liver inflammation and damage. As the liver is thought to be a major site for cytokine production in shock, selectin-based therapy may not be useful in preventing systemic damage induced during shock when the liver is involved. These data may also be relevant to designing therapies for liver injury associated with transplantation or various forms of hepatitis. On the other hand, the lack of effect of selectin-based therapy in organs such as the liver may be advantageous when targeting specific skin diseases or reperfusion injury to the intestine or skeletal muscle wherein impairment of leukocyte recruitment into the liver may be unnecessary or even detrimental. Finally, these data may extend to other organs including the lung microvasculature. Much like the liver, leukocyte adhesion occurs primarily in the capillaries in the pulmonary circulation and leukocyte recruitment may also bypass the selectin pathway in this organ (39).

Clearly, the work accomplished to date in the mesentery, cremaster, and cheek pouch, as well as in in vitro laminar flow chambers, has been essential to our understanding of leukocyte recruitment under relatively high shear conditions in postcapillary venules. However, under the hydrodynamic conditions found in the hepatic sinusoids and based on the architecture of these vessels, the selectins may be of little consequence in leukocyte recruitment to this organ. Intravital microscopy assessment in organs such as the lung, kidney, heart, and other major organs is clearly warranted. An intriguing point is that leukocytes adhere in the capillaries of liver (sinusoids) but not the cremaster microvasculature. Whether this is related to the differential expression of adhesion molecules within these vessels will be an important issue to investigate. In fact, it may be predictive of which other vascular beds will permit adhesion within the capillaries and thereby perhaps bypass the necessity for selectins in the leukocyte recruitment process.

\section{Acknowledgments}

This work was supported by the Canadian Medical Research Council (HT 11537) and the National Institutes of Health (grants AI 19031 and ES 06091) P. Kubes is an Alberta Heritage Foundation Medical Research Scholar and a Medical Research Council scientist.

\section{References}

1. Kubes, P., and S. Kanwar. 1994. Histamine induces leukocyte rolling in post-capillary venules: a P-selectin-mediated event. J. Immunol. 152:3570-3577.
2. Kubes, P., M.A. Jutila, and D. Payne. 1995. Therapeutic potential of inhibiting leukocyte rolling in ischemia/reperfusion. J. Clin. Invest. 95:2510-2519.

3. Mayadas, T.N., R.C. Johnson, H. Rayburn, R.O. Hynes, and D.D. Wagner. 1993. Leukocyte rolling and extravasation are severely compromised in P-selectin-deficient mice. Cell. 74:541-554.

4. Ley, K., D.C. Bullard, M.L. Arbones, R. Bosse, D. Vestweber, T.F. Tedder, and A.L. Beaudet. 1995. Sequential contribution of L- and P-selectin to leukocyte rolling in vivo. J. Exp. Med. 181:669-675.

5. Finger, E.B., K.D. Puri, R. Alon, M.B. Lawrence, U.H. Von Andrian, and T.A. Springer. 1996. Adhesion through L-selectin requires a threshold hydrodynamic shear. Nature (Lond.). 379:266-269.

6. Von Andrian, U.H., J.D. Chambers, L.M. McEvoy, R.F. Bargatze, K.E. Arfors, and E.C. Butcher. 1991. Two-step model of leukocyte-endothelial cell interaction in inflammation: distinct roles for LECAM-1 and the leukocyte $\beta_{2}$ integrins in vivo. Proc. Natl. Acad. Sci. USA. 88:7538-7542.

7. Ley, K., P. Gaehtgens, C. Fennie, M.S. Singer, L.A. Lasky, and S.D. Rosen. 1991. Lectin-like cell adhesion molecule 1 mediates leukocyte rolling in mesenteric venules in vivo. Blood. 77:2553-2555.

8. Lindbom, L., X. Xie, J. Raud, and P. Hedqvist. 1992. Chemoattractantinduced firm adhesion of leukocytes to vascular endothelium in vivo is critically dependent on initial leukocyte rolling. Acta. Physiol. Scand. 146:415-421.

9. Jaeschke, H., A. Farhood, and C.W. Smith. 1990. Neutrophils contribute to ischemia/reperfusion injury in rat liver in vivo. FASEB (Fed. Am. Soc. Exp. Biol.) J. 4:3355-3359.

10. Holman, J.M. and T.M. Saba. 1988. Hepatocyte injury during postoperative sepsis: activated neutrophils as potential mediators. J. Leukocyte Biol. 43: 193-203.

11. Jaeschke, H., A. Farhood, A.P. Bautista, Z. Spolarics, J.J. Spitzer, and C.W. Smith. 1993. Functional inactivation of neutrophils with a Mac-1 (CD11b/ CD18) monoclonal antibody protects against ischemia-reperfusion injury in rat liver. Hepatology. 17:915-923.

12. Essani, N.A., M.A. Fisher, A. Farhood, A.M. Manning, C.W. Smith, and H. Jaeschke. 1995. Cytokine-induced upregulation of hepatic intercellular adhesion molecule-1 messenger RNA expression and its role in the pathophysiology of murine endotoxin shock and acute liver failure. Hepatology. 21:16321639.

13. Steinhoff, G., M. Behrend, B. Schrader, and R. Pichlmayr. 1993. Intercellular immune adhesion molecules in human liver transplants: overview on expression patterns of leukocyte receptor and ligand molecules. Hepatology. 18:440-453.

14. Essani, N.A., G.M. McGuire, A.M. Manning, and H. Jaeschke. 1995. Differential induction of mRNA for ICAM-1 and selectins in hepatocytes, Kupffer cells and endothelial cells during endotoxemia. Biochem. Biophys. Res. Commun. 211:74-82.

15. Dore, M., H.K. Hawkins, M.L. Entman, and C.W. Smith. 1996. Production of a monoclonal antibody against canine GMP-140 (P-selectin) and studies of its vascular distribution in canine tissues. Vet. Pathol. 30:213-222.

16. Adams, D.H., P. Burra, S.G. Hubscher, E. Elias, and W. Newman. 1994. Endothelial activation and circulating vascular adhesion molecules in alcoholic liver disease. Hepatology. 19:588-594.

17. Bullard, D.C., L. Qin, I. Lorenzo, W.M. Quinlin, N.A. Doyle, C.M. Doerschuk, and A.L. Beaudet. 1995. P-selectin/ICAM-1 double mutant mice: acute emigration of neutrophils into the peritoneum is completely absent but is normal into pulmonary alveoli. J. Clin. Invest. 95:1782-1788.

18. Bullard, D.C., E.J. Kunkel, H. Kubo, M.J. Hicks, I. Lorenzo, N.A. Doyle, C.M. Doerschuk, K. Ley, and A.L. Beaudet. 1996. Infectious susceptibility and severe deficiency of leukocyte rolling and recruitment in E-selectin and P-selectin double mutant mice. J. Exp. Med. 183:2329-2336.

19. Nishida, J., R.S. McCuskey, D. McDonnell, and E.S. Fox. 1994. Protective role of nitric oxide in hepatic microcirculatory dysfunction during endotoxemia. Am. J. Physiol. 267:G1135-G1141.

20. McCuskey, R.S. 1986. Microscopic methods for studying the microvasculature of internal organs. In Physical Techniques in Biology and Medicine Microvascular Technology. C.H. Barker and W.F. Nastuk, editors. Academic Press Inc., Orlando, FL. 247-264.

21. Ley, K., G. Linnermann, M. Meinen, L.M. Stoolman, and P. Gaehtgens. 1993. Fucoidin, but not yeast polyphosphomannan PPME, inhibits leukocyte rolling in venules of the rat mesentery. Blood. 81:177-185.

22. Gaboury, J.P., B. Johnston, X.F. Niu, and P. Kubes. 1995. Mechanisms underlying acute mast cell-induced leukocyte rolling and adhesion in vivo. J. Immunol. 154:804-813.

23. Vollmar, B., M.D. Menger, J. Glasz, R. Leiderer, and K. Messmer. 1994. Impact of leukocyte-endothelial cell interaction in hepatic ischemia-reperfusion injury. Am. J. Physiol. 267:G786-G793.

24. Henninger, D.W., J. Panes, M. Eppihimer, J. Russell, M. Gerritsen, D.C. Anderson, and D.N. Granger. 1997. Cytokine-induced VCAM-1 and ICAM-1 expression in different organs of the mouse. J. Immunol. 158:18251832.

25. Gaboury, J.P., and P. Kubes. 1994. Reductions in physiologic shear rates lead to CD11/CD18-dependent, selectin-independent leukocyte rolling in vivo. Blood. 83:345-350.

26. Arndt, H., P. Kubes, M.B. Grisham, E. Gonzalez, and D.N. Granger. 
1992. Granulocyte turnover in the feline intestine. Inflammation. 16:549-559.

27. Asako, H., R.E. Wolf, and D.N. Granger. 1993. Leukocyte adherence in rat mesenteric venules: effects of adenosine and methotrexate. Gastroenterology. 104:31-37.

28. Sririmarao, P., U.H. Von Andrian, E.C. Butcher, M.A. Bourdon, and D.H. Broide. 1994. L-selectin and very late antigen-4 promote eosinophil rolling at physiologic shear rates in vivo. J. Immunol. 153:4238-4246.

29. Bienvenu, K., J. Russell, and D.N. Granger. 1992. Leukotriene $B_{4}$ mediates shear rate-dependent leukocyte adhesion in mesenteric venules. Circ. Res. 71:906-911.

30. Bienvenu, K., J. Russell, and D.N. Granger. 1993. Platelet-activating factor promotes shear rate-dependent leukocyte adhesion in postcapillary venules. J. Lipid Mediators. 8:95-103.

31. Rosengren, S., A.M. Olofsson, U.H. Von Andrian, E. Lundgren-Akerlund, and K.E Arfors. 1991. Leukotriene $\mathrm{B}_{4}$-induced neutrophil-mediated endothelial leakage in vitro and in vivo. J. Appl. Physiol. 71:1322-1330.

32. Bjork, J., L. Lindbom, B. Gerdin, G. Smedegard, K.E. Arfors, and J. Benveniste. 1983. PAF-acether (platelet-activating factor) increases microvascular permeability and affects endothelium-granulocyte interaction in microvascular beds. Acta Physiol. Scand. 119:305-308.

33. Dahlen, S.E., J. Bjork, P. Hedqvist, K.E. Arfors, S. Hammarstrom, J.A. Lindgren, and B. Samuelsson. 1981. Leukotrienes promote plasma leakage and leukocyte adhesion in postcapillary venules: in vivo effects with relevance to the acute inflammatory response. Proc. Natl. Acad. Sci. USA. 78:3887-3891.

34. Labow, M.A., C.R. Norton, J.M. Rumberger, K.M. Lombard-Gillooly, D.J. Shuster, J. Hubbard, R. Bertko, P.A. Knaack, R.W. Terry, M.L. Harbison, et al. 1994. Characterization of E-selectin-deficient mice: demonstration of overlapping function of the endothelial selectins. Immunity. 1:709-720.

35. Kanwar, S., D.C. Bullard, M.J. Hickey, C.W. Smith, A.L. Beaudet, B.A Wolitzky, and P. Kubes. 1997. The association between $\alpha 4$-integrin, P-selectin, and E-selectin in an allergic model of inflammation. J. Exp. Med. 185:10771087.

36. Frenette, P.S., T.N. Mayadas, H. Rayburn, R.O. Hynes, and D.D. Wagner. 1996. Susceptibility to infection and altered hematopoiesis in mice deficient in both P- and E-selectins. Cell. 84:563-574.

37. Kvietys, P.R., B. Twohig, J. Danzell, and R.D. Specian. 1990. Ethanolinduced injury to the rat gastric mucosa. Role of neutrophils and xanthine oxidase-derived radicals. Gastroenterology. 98:909-920.

38. Kunkel, E.J., U. Jung, D.C. Bullard, K.E. Norman, B.A. Wolitzky, D. Vestweber, A.L. Beaudet, and K. Ley. 1996. Absence of trauma-induced leukocytes rolling in mice deficient in both P-selectin and intercellular adhesion molecule 1. J. Exp. Med. 183:57-65.

39. Mizerd, J.P., B.B. Meek, G.J. Kutkoski, D.C. Bullard, A.L. Beaudet, and C.M. Doerschuk. 1996. Selectins and neutrophil traffic: margination and Streptococcus pneumoniae-induced emigration in murine lungs. J. Exp. Med. 184:639-645. 